\title{
Eine Ars moriendi für unsere Zeit
}

\author{
Brigitte Hopp
}

\author{
Selbst bereits im fortgeschrittenen Alter, macht sich die Autorin Gedanken über \\ Sterben, Tod und die damit verbundenen Ängste. Geboren 1923, studierte sie im \\ Nachkriegsdeutschland Medizin und absolvierte eine Facharztausbildung in Psy- \\ chiatrie und Neurologie. 1958 siedelte sie in die Schweiz über, wo sie an verschie- \\ denen kantonalen Kliniken arbeitete, ab 1970 im ehemaligen Sanatorium Bins- \\ wanger als Leiterin einer stationären Psychotherapie-Gruppe.
}

\section{An den Rand gedrängt - verdrängt}

In unserer Gesellschaft erleben wir, dass das Thema Tod bei vielen Menschen mit Ängsten besetzt ist, wobei nicht nur der Sterbevorgang an sich eine Rolle spielt - was verständlich ist, da nicht immer berechenbar -, sondern auch der Fortgang nach dem Todesereignis. Oft ist sogar der Umgang mit dem toten Körper, auch wenn es sich um einen geliebten Menschen handelt, tabuisiert. Das müsste nicht so sein. In früheren Zeiten gingen die Menschen mit dem Tod freier und natürlicher um. Neben den Kirchen fand man oft Beinhäuser, in denen die Gebeine der früher verstorbenen Gemeindemitglieder aufbewahrt und meistens gut sichtbar ausgestellt waren. Das Memento mori war im kirchlichen Raum an vielen Orten präsent. Und da die Menschen viel weniger alt wurden, war der Tod auch im familiären Erleben gegenwärtiger. Er war in das Leben eingebunden. Durch den Fortschritt der Medizin ist der Tod immer mehr an den Rand des Lebens und auch des Bewusstseins gedrängt worden. Das Leben ist diesseitiger geworden und die religiöse Bindung hat sich in weiten Teilen der Bevölkerung gelockert. Es scheint so, als sei die Angst vor dem Sterben an sich dadurch gestiegen.

Bei Kindern indessen ist zu beobachten, dass sie im Vorschul- und Schulalter dem Tod ohne grosse Angst begegnen, sofern er nicht mit Gewaltsituationen verbunden ist. Sie fragen vielfach ganz sachlich, wo denn nun der gestorbene Mensch sei, wie es ihm wohl gehe, ob er einmal wieder zurückkehre.

Im Erwachsenenalter ist diese kindlich-einfache Perspektive nicht mehr gegeben. Wir erleben die Welt mit all ihren Ungereimtheiten. Das Leben wird komplizierter: Wir haben Gebote und Verbote erfahren. Wir haben sie übertreten oder befolgt. Wir wurden gelobt und getadelt - vielleicht sogar bedroht. Wir rebellierten gegen Eltern und Staat und vielleicht eine Reihe anderer Autoritäten. Wir fühlten uns stolz und stark dabei oder wir hatten ein schlechtes Gewissen ob kleiner oder auch grosser Verfehlungen. Unter günstigen Bedingungen reifen wir, entwickeln unser Gewissen und werden in diesem Prozess sicherer, stärker und realitätsbewusster. Im ungünstigen Fall werden wir verängstigt und bedrückt oder auch hochfahrend, vielleicht sogar bösartig. Wir werden älter, und es gibt so manchen Geburtstag - auch sogenannte «runde». Manchmal hinterfragen wir kritisch

\section{«Das Memento mori war im kirchlichen Raum} an vielen Orten präsent.»

das bisherige oder zukünftige Leben: Habe ich eine Ausbildung durchlaufen, die meinem Wesen und meinen Möglichkeiten entsprochen hat? Bin ich so erfolgreich gewesen, wie ich es sein wollte? Hätte ich nicht viel mehr erreichen müssen? Bin ich nicht zu oft zu nachgiebig oder zu nachlässig gewesen? War ich meinem Partner und meiner Verwandtschaft gegenüber ein treuer Mensch? Alle diese Fragen drängen sich in der Mitte des Lebens häufig in den Vordergrund. Auch kann der Tod urplötzlich und verstörend in das eigene Leben einbrechen, indem zum Beispiel nahe Verwandte oder Freunde unverhofft sterben.

\section{Begrenzte Lebenszeit wie nutzen?}

Von vielen dieser Sorgen und Ängste kann man sich durch eine entsprechende, auf den Tod als das Endziel des Lebens bezogene Lebensplanung und -führung freihalten. Ist mir bewusst, dass meine Lebenszeit begrenzt ist, dass ich sie nutzen und am Lebensziel arbeiten sollte? Wie viel Erholung, Ver- 
gnügen, Spass soll oder darf sein? Bin ich mit mir selbst und meinem Leben im Reinen? Stehe ich mit Freude und gutem Gefühl im Leben? Sind meine mitmenschlichen Beziehungen in Ordnung oder bereinigt? Was ist dazu noch zu tun? Wo habe ich Schulden? Wo Defizite verschiedener Art?

Damit eine solche Lebensplanung und -führung gelingen kann, müssen die Grundlagen bereits in der Familie durch die Eltern gelegt werden. Im weiteren Verlauf der Entwicklung haben auch Kindergarten und Schule ihren Beitrag zu leisten. Schliesslich sind auch die Kulturschaffenden und die Medien gefordert, sich mit dieser Thematik zu beschäftigen, um damit ihrer Verantwortung der Gesellschaft gegenüber gerecht zu werden.

«Da die Menschen viel weniger alt wurden, war der Tod auch im familiären Erleben gegenwärtiger.»

Mit einer solchen Einstellung, die den Tod als Endpunkt des Lebens nicht aus den Augen verliert, kann auch der letzte Lebensabschnitt ohne Angst angegangen werden. Wir müssen rechtzeitig, das heisst, solange wir noch selbst entscheidungsfähig sind, unsere persönlichen und überpersönlichen Angelegenheiten regeln. Dazu gehören zum Beispiel die Abfassung einer Patientenverfügung und die Rege- lungen für die letzten Jahre, Monate oder Wochen des Lebens. Die meisten Menschen wünschen sich ja, in ihrer eigenen vertrauten Umgebung zu sterben. Deshalb muss die spitalexterne Kranken- und Alterspflege entsprechend ausgebaut werden. Idealerweise sind auch Familienangehörige oder andere vertraute Menschen in der Lage, den Sterbenswilligen Hilfe und Begleitung zu bieten.

\section{Natürlich sterben}

Ausdrücklich möchte ich mich vom Suizid abgrenzen, wie er zum Teil von älteren und einsamen Menschen angestrebt wird. Er ist nur selten eine Tat der äussersten Verzweiflung, sondern vielfach eine

Demonstration: «Ihr habt mich nicht verstanden.» Oder: «Du, mein Freund, hast mich nicht genug geliebt.» Oder: «Ihr habt gar nicht gemerkt, wie schlecht es mir geht. Jetzt seht ihr es!»

Ebenso deutlich grenze ich mich auch von «Exit» und ähnlichen Organisationen ab, deren Dienste zu meinem grossen Erstaunen auch ein bekannter katholischer Theologe in Anspruch nehmen will. Ich möchte nicht ein Medikament nehmen, mit dem ich bequem den Tod überstehen, aber nicht erleben kann. Der mehr und mehr lockere Umgang vieler Menschen mit dem Tod erfüllt auch mich mit Besorgnis. Der

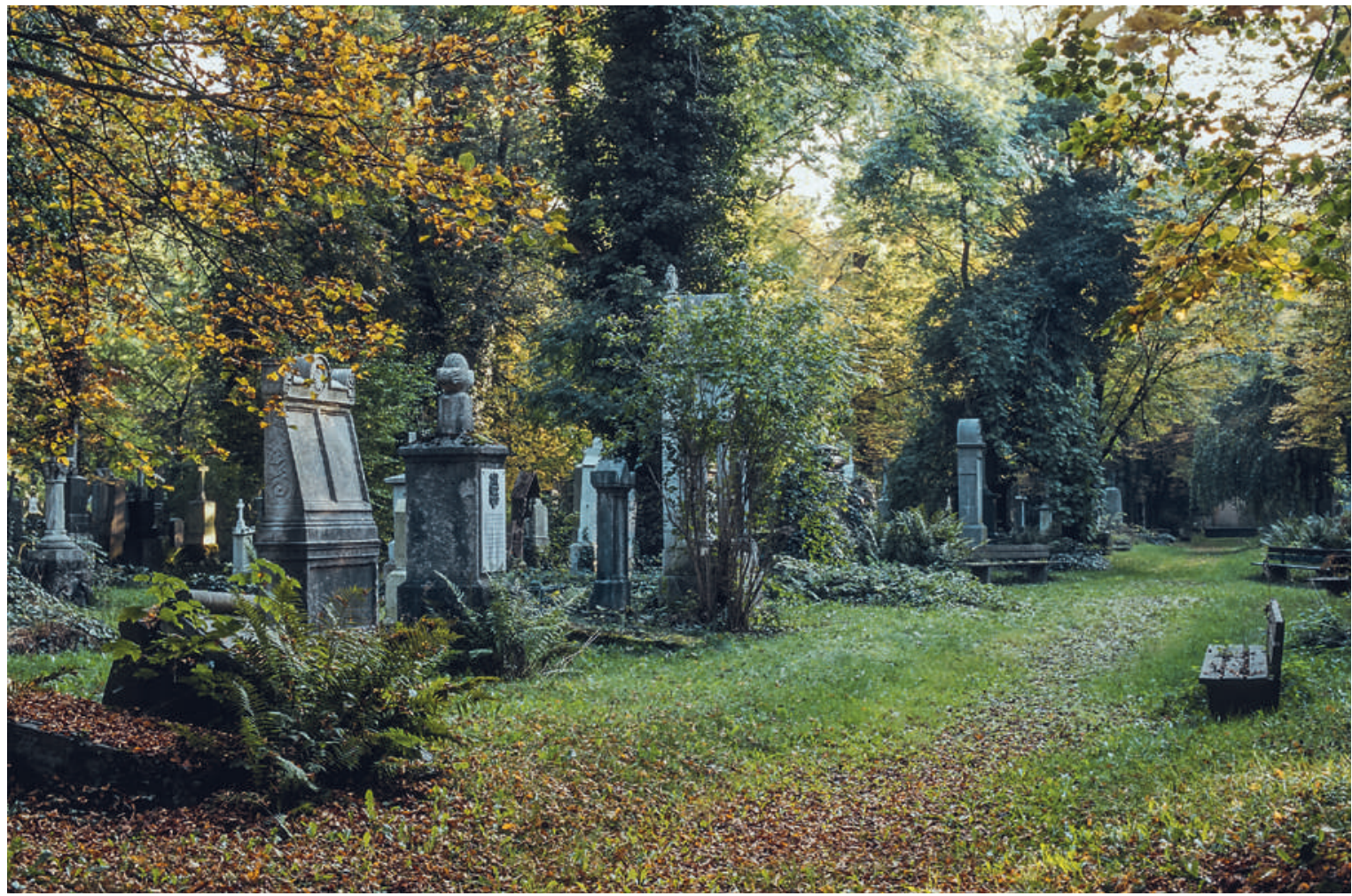

Eine Lebensplanung und -führung, die das unausweichliche Ende miteinbezieht, kann von Sorgen und Angst vor dem Tod befreien. 
Tod ist keine einfache Sache. Es ist falsch, den Sterbevorgang einfach mit Hilfe einer Pille zu lenken, zu beenden und möglicherweise sogar zu inszenieren. Es läuft dem christlichen Glauben zuwider, der uns lehrt, unser Leben sei von Beginn an, also von der Verbindung von Samen und Eizelle an, in Gottes Hand, und in dieses Leben einzugreifen sei eine sündige Handlung, sowohl als Eingriff in fremdes als auch in das eigene Leben. Jedoch hat Kant uns auch gelehrt, dass es das Gute an sich nicht gibt, sondern nur den guten Willen. So kann Sterbehilfe bei unheilbar kranken Menschen in Einzelfällen gerechtfertigt sein, wenn dadurch ein schreckliches, für ein Individuum nicht mehr erträgliches Leiden abgewendet werden kann.

Jedoch zurück zur oben angesprochenen Sterbehilfe. Irgendwann kommt die Zeit der immer unmittelbareren Todesnähe, die meistens von den Betroffenen mehr und mehr wahrgenommen wird und nicht immer - aber oft - auch gerne mit Freunden oder Familienangehörigen besprochen werden möchte. Wichtig ist es, diese Gespräche ohne Scheu oder Scham zuzulassen, ihnen ein ernstes Verständnis entgegenzubringen. So wird sowohl das Sterben als auch die Trauerarbeit erleichtert. Ist die Zeit der unmittelbaren Todesnähe gekommen, wo eine Verzögerung des Todes keine neuen Gesichtspunkte mehr bringt, wo ich nichts mehr bewegen kann, sondern der Zeitpunkt gekommen ist, wo ich mich in die behütenden Arme des "Gevatters Tod» oder des «Freundes Hein» fallen lassen möchte - nicht aus Angst vor dem Tod oder dem Leben, sondern aus der Erkenntnis und dem Gefühl heraus, dass mein Leben seinen natürlichen Abschluss finden sollte, dann nehme ich keine lebenserhaltenden Medikamente mehr ein, esse nur noch, wenn ich unbedingt möchte. Natürlich werde ich schwächer. Hunger und Durst merke ich nicht mehr. Ich trinke nicht mehr und schlafe in der Regel im Laufe einiger Tage ruhig ein. Dank diesem Vorgehen kommt es nur selten zu einer Phase ängstlicher Erregung, die mit entsprechenden beruhigenden Medikamenten und einer einfühlsamen Begleitung gemildert oder behoben werden kann. Die oft ausgetrockneten und daher schmerzhaften Schleimhäute in Nase, Mund und Rachen kann man befeuchten und dadurch dem Sterbenden eine grosse Erleichterung verschaffen. Es kann auch nötig sein, starke Schmerzen zu bekämpfen. Ärzte dürfen die Schmerztherapie durchführen, auch wenn dadurch das Leben um Tage oder Stunden verkürzt werden könnte. So kann man sich ein natürliches Sterben vorstellen, das aus eigenem Willen erfolgt, aber die Gefühle der Angehörigen nicht verletzt.
Wie alles Lebendige auf unserer Erde ist auch diese Vorbereitung auf ein würdiges Sterben abhängig von der Persönlichkeit des betreffenden Menschen. Von dem banalen Stolz, alles ins Reine gebracht zu haben, über ein Pflichtgefühl sich selbst und der persönlichen Umwelt gegenüber bis zum freudigen und freiheitlichen Empfinden der Erwartung des Kommenden. Dieses letztere Gefühl hat mich selbst seiner Zeit jeweils vor der Geburt meiner Kinder, wenn die Wehen einsetzten, begleitet und sie dadurch schmerzarm und freudig-erwartungsvoll gemacht. Und so hoffe ich selbst auch, meinen letzten Weg gut bewältigen zu können.

«Ist mir bewusst, dass meine Lebenszeit begrenzt ist, dass ich sie nutzen und am Lebensziel arbeiten sollte?»

Das seelische Geschehen klammert die materielle Realität häufig nicht aus. In so manchem Todesfall ist die Zeit der Todesnähe durch Krankheiten geprägt, die durch oft sinnlose, weil nicht mehr wirksame ärztliche Massnahmen verlängert werden. Dadurch wird der natürliche Sterbevorgang künstlich aufgehalten und eigentlich gestört. Es besteht die Gefahr, dass Familien in finanzielle Notlagen geraten und Krankenkasse und Sozialsysteme immens belastet werden. Dieser wirtschaftliche Aspekt gewinnt in der öffentlichen Diskussion zunehmend an Bedeutung. Deswegen sollte die Entwicklung hin zu einer guten Betreuung in der letzten Lebenszeit gehen und so weit wie möglich sinnlose ärztliche Leistungen ausschliessen. Die Differenzierung zwischen sinnvollen und sinnlosen Massnahmen ist selten schwierig: Was der momentanen Erleichterung des Leidens dient, kann sinnvoll sein, was auf die Verlängerung des Lebens abzielt, ist es nicht.

Das Hinübergleiten vom Leben hier in das Nachher muss nicht von Ängsten begleitet sein und kann in vielen Fällen selbstbestimmt erfolgen, ohne auf «Exit» oder ähnliche Dienstleistungen zurückgreifen zu müssen. Wir kennen das Nachher zwar nicht und machen uns je nach Religion und Weltanschauung verschiedene Vorstellungen davon. Was uns aber wirklich erwartet, weiss niemand genau. Vielleicht ist es eine Abrechnung mit dem Leben, vielleicht verbunden mit leidvollen Erkenntnissen, vielleicht ist es die ewige Ruhe.

\footnotetext{
Literatur

- Brigitte Hopp. Psychotherapie. Meine persönlichen Wege zur Heilung seelischer Störungen. Borsdorf: Verlag winterwork. ISBN 978-3864682124.
} 